\title{
Ein kritischer Blick auf Angelika Neuwirths Koranverständnis
}

\author{
Dr. Tarek Elkot \\ Abteilung für Islamische Studien in \\ Fremdsprachen \\ Sektion für Islamische Studien in Deutsch \\ Fakultät für Fremdsprachen und Translatologie, \\ Al-Azhar Universität, Kairo/Ägypten
}


(ISSN : 2536 - 9555)

\title{
A critical viewpoint of Angelika Neuwirth's
}

\author{
understating of the Qur'an
}

\section{Dr. Tarek Elkot}

\section{Department of Islamic Studies in Foreign Languages (German section), Faculty of Languages and Translation, Al-Azhar University, Cairo, Egypt.}

\begin{abstract}
English:
Through presentation, analysis and criticism, the research discusses the modern tendency of Qur'anic studies in Europe, which views the Qur'an as a text expressing the community's culture. This trend is represented in Germany by Professor of Islamic Studies Angelika Neuwirth and her project (Corpus Coranicum) that she launched in 2007 at the BerlinBrandenburg Academy of Sciences in Germany.

In her various studies and articles on the Qur'an, Neuwirth tries repeatedly to assert that the Qur'an is not an authored text. She rejects the idea that the Qur'an is attributed only to the Prophet Muhammad, such as representatives of traditional European Qur'anic research have said. For her, the Qur' an is a text that was contributed to by various actors. According to Neuwirth, the community surrounding the Prophet Muhammad plays a very important role. Therefore, Neuwirth does not consider the Qur'an to be a document of one person, but rather a document expressing the culture of the community.

According to Neuwirth, the community surrounding Muhammad is considered a Jewish and Christian society.
\end{abstract}


Consequently, the Qur'an contains both Jewish and Christian traditions, which nevertheless were not adopted directly. Rather, the Prophet Muhammad and the community around him had a role in treating them. According to Neuwirth's view, the Qur'an contains not only Jewish and Christian traditions, but also pagan traditions.

According to this understanding, the role of the first community is not limited to discuss with the Prophet, but also to the negative co-authoring of the Qur'an. The group, along with the Prophet Muhammad, participated in the compilation of the Qur'an.

From the above, it results that no difference between traditional and modern European research on the Qur'an with regard to understanding the origin of the Qur'an. Neuwirth agrees with representatives of traditional Qur'anic scholarship in denying the Qur'an the divine origin.

\section{Keywords:}

The Qur'an, Qur'anic Studies in Europe, Angelika Neuwirth, the first islamic community. 
(ISSN : 2536 - 9555)

\section{نظرة نقدية لتصورات أنجليكا نويفرت عن القرآن}

\section{د. طارق أنور عبد الجيد القط}

قسم الدراسات الإسلامية باللفات الأجنبية، شعبة ألماني، كلية اللفات الترجمة،

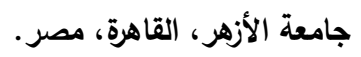

الملخص باللغة العربية:

يناقش البحث من خلال العرض والتحليل والنقد الاتجاه الحديث للدراسات القرآنية

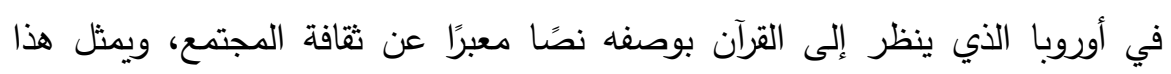

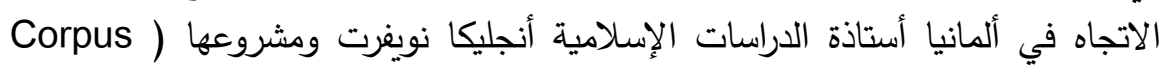

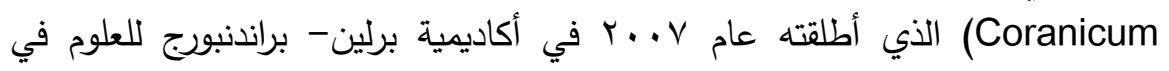
ألمانيا.

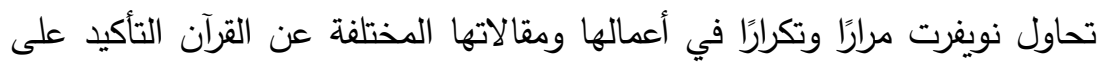

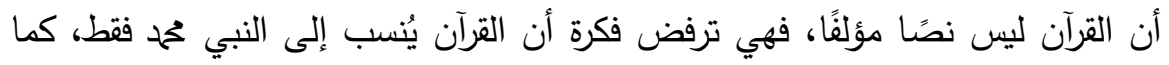

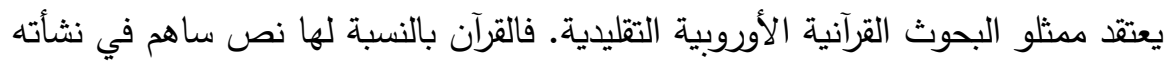

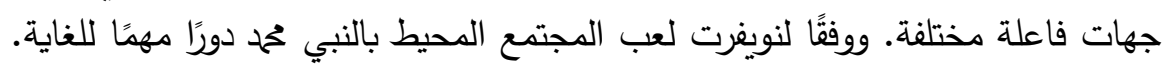
لذلك لا تعتبر نويفرت القرآن وثيقة لشخص واحدة لثند ولكنه وثيقة معبرة عن ثقافة المجتمع.

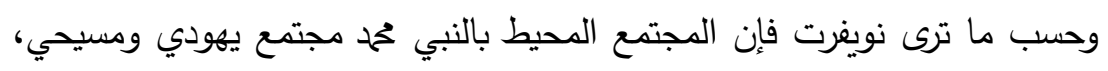
ولهذا السبب فالقرآن يحتوي على تقاليد يهودية ومسيحية، والتي مع ذلك لم لم يتم تبنيتها بشكل مباشر، بل كان للنبي ححمد والمجتمع من حوله دور في معالجتها. وطبقا لما تراه نويفرت فلا يحتوي القرآن على تقاليد يهودية ومسيحية فقط، بل على بلى تقاليد وثنية أيضًا.

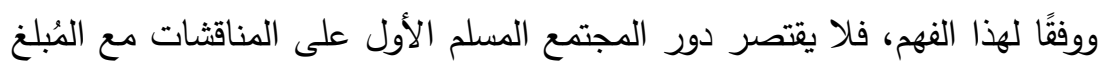

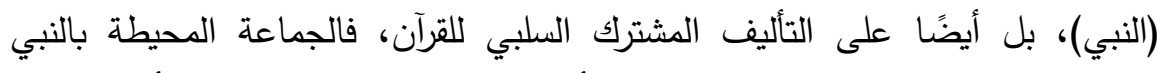

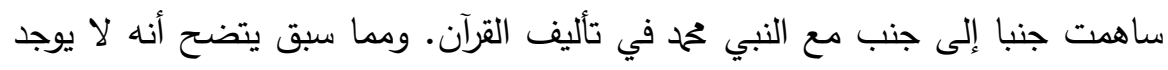

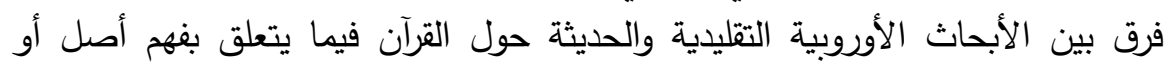

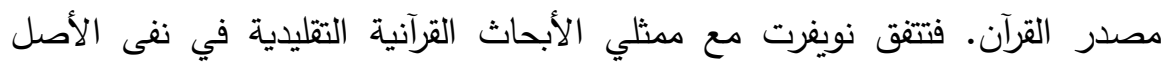
الإلهي عن القرآن.

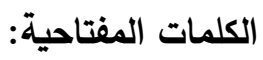

القرآن، الدراسات القرآنية في أوروبا، أنجليكا نويغرت، مجتمع المسلمين الأوائل. 
Ein kritischer Blick auf Angelika Neuwirths Koranverständnis

Dr. Tarek lkot

مجلة وادي النيل للاراسات والبحوث الإنسانية والاجتماعية والتزبوية (مجلة علمية محكمة)

Ein kritischer Blick auf Angelika Neuwirths Koranverständnis

Dr. Tarek Elkot

\section{Einleitung}

In der Beschäftigung mit dem Koran rückt die europäische Koranforschung den Propheten Muhammad in den Vordergrund. Im Gegensatz zur traditionellen europäischen Koranforschung, die sich für Muhammad als den einzigen Akteur für die Entstehung des Korans ausspricht, steht die jüngste europäische Koranforschung, in der von verschiedenen Akteuren die Rede ist. Muhammad ist nicht mehr als der alleinige Akteur in der Konstitution des Korantextes anzusehen. Die Gemeinde, an die die Verkündigung gerichtet werde, gelte dabei als ein wichtiger Akteur. Die Diskussionen zwischen Verkünder und Gemeinde würden sich im Koran widerspiegeln. ${ }^{1}$

In seinem Buch Gottes Offenbarung in Menschenwort, Der Koran im Licht der Barmherzigkeit spricht Mouhand Khourchide unter anderem über das Verständnis des Korans als eine offene vom historischen Kontext des 7. Jahrhunderts geprägte Kommunikation. In diesem Zusammenhang verweist er auf die deutsche Islamwissenschaftlerin Angelika Neuwirth und schreibt Folgendes: „Angelika Neuwirth unterstreicht zu Recht, dass der Koran das Zeugnis einer Gemeindebildung im 7. Jahrhundert darstellt. “2 Der Koran spiegelt nach dieser Vorstellung sowohl den historischen Kontext als auch die Gemeindebildung des 7. Jahrhunderts wider.

${ }^{1}$ Würsch, Renate: Der Koran und seine Rezeption, in: Das Mittelalter 2018 (2013) 1, S. 27-45, hier S. 37.

2 Khorchide, Mouhand: Gottes Offenbarung in Menschenwort, Der Koran im Licht der Barmherzigkeit, Herder Verlag, Freiburg 2018, S. 84. 
(ISSN : 2536 - 9555)

Der Koran ist nach Ömer Özsoy „als historisches Dokument einer beweglichen Gemeindebildung anzusehen" ${ }^{3}$ Er besteht aus Offenbarungseinheiten, „die auf verschiedene Sprechakte verweisen, an denen jeweils unterschiedliche Akteure mit verschiedenen Wissenshintergründen beteiligt waren". ${ }^{4}$ Özsoy bestätigt dieselbe Idee, der zufolge der Koran für Dokument einer Gemeindebildung zu erklären ist. Die verschiedenen Akteure mit ihren verschiedenen Wissenshintergründen beteiligten sich an der Entwicklung des Korantextes. In diesem Zusammenhang stellt man die Frage nach diesen Wissenshintergründen und ihren Einflüssen auf den Koran.

Neuwirth, auf deren Ideen über den Koran als Text der Gemeindebildung die Studie fokussiert, versteht den Koran nicht „als einen Text der islamischen Tradition, sondern historisch, als Dokument einer Gemeindebildung inmitten einer christlichjüdisch-synkretistisch geprägten Debattenlandschaft“ ${ }^{5}$ In ihrem ersten Band Der Koran, Bd. 1: Frühmekkanische Suren, Poetische Prophetie, Handkommentar mit Übersetzung von Angelika Neuwirth erläutert Neuwirth das Ziel, indem sie Folgendes sagt:

„In diesem ersten Band des historischliteraturwissenschaftlichen Handkommentars zum Koran wird erstmals der Versuch unternommen, den Koran als fortlaufende Verkündigung zu beschreiben und ihn so als textuellen Niederschlag einer Gemeindebildung aus der Neureflexion älterer biblischer und nachbiblischer

3 Özsoy, Ömer: Das Unbehagen der Koranexegese - Den Koran in anderen Zeiten zum Sprechen bringen, in: Kontexte, Methoden, Inhalte, Frankfurter Zeitschrift für Islamisch-theologische Studien, EB-Verlag, Berlin 2014, S. 43.

${ }_{5}^{4}$ Ebd., S. 43.

5 Neuwirth, Angelika: Der Koran als Text der Spätantike. Ein europäischer Zugang, 1. Aufl., Verlag der Weltreligionen, Berlin 2010, S. 15. 
Ein kritischer Blick auf Angelika Neuwirths Koranverständnis

Dr. Tarek lkot

مجلة وادي النيل للاراسات والبحوث الإنسانية والاجتماعية والتربوية (مجلة علمية محكمة)

Traditionen wie auch des altarabischen poetischen Erbes zu deuten." ${ }^{\text {66 }}$

Die Gemeinde spiegelte sich im Korantext „nicht nur als Empfängerschaft, sondern als ein Kollektiv von Mitsprechern, als Frager, als Konsensbildende" ${ }^{\text {" } 7}$, so schreibt auch Neuwirth auch. Es gibt keinen Zweifel daran, dass die Gemeinde um den Propheten Muhammad nicht nur Empfängerin, sondern auch Fragende ist. Eine Reihe von Koranversen beginnt mit an den Propheten Muhammad gerichteten Fragen. Man soll hier aber darauf aufmerksam machen, dass die mit Fragen beginnenden Koranverse nur einen geringen Teil des Korans darstellen.

Immer wieder deutet Neuwirth in ihren verschiedenen Werken und Artikeln auf eine enge Verbindung zwischen Koran und Bildung der Gemeinde hin. Im Gegensatz zur traditionellen europäischen Koranforschung ist sie nicht davon überzeugt, dass der Koran die Horizonte bzw. die Hintergründe einer einzelnen Person zum Ausdruck bringt. „Der Koran ist Ausdruck nicht der Überzeugung eines einzelnen, sondern eines sich sukzessiv bildenden Gemeindekonsenses. " ${ }^{8}$ Das heißt, dass sich der Korantext immer wieder mit der Entwicklung der Horizonte der Gemeinde entwickelt. Der Text bezeugt daraufhin die sich herausbildende Debattenkultur der Gemeinde.

Die oben genannten Ausführungen weisen die neue europäische Richtung zum Umgang mit dem Koran auf. Dabei wird von neuen

${ }^{6}$ Neuwirth, Angelika: Der Koran, Bd. 1: Frühmekkanische Suren, Poetische Prophetie, Handkommentar mit Übersetzung von Angelika Neuwirth, 1. Aufl., Verlag der Weltreligionen, Berlin 2011, S. $22-23$.

${ }^{7}$ Neuwirth, Angelika: Koranforschung - eine politische Philologie? Bibel, Koran und Islamentstehung im Spiegel spätantiker Textpolitik und moderner Philologie, De Gruyter, Berlin 2014, S. 14.

${ }^{8}$ Neuwirth, Angelika: Der Koran, Bd. 2/1: Frühmittelmekkanische Suren, Das neue Gottesvolk: Biblisierung des altarabischen Weltbildes, Handkommentar mit Übersetzung von Angelika Neuwirth, 1. Aufl., Verlag der Weltreligionen, Berlin 2017, S. 36. 
(ISSN : 2536 - 9555)

Faktoren gesprochen, die einen wichtigen Beitrag zur Entwicklung des Korantextes leisteten. $\mathrm{Zu}$ diesen neuen Faktoren gehören vor allem die Gemeinde, die Gemeindebildung und die unterschiedlichen Akteure mit ihren verschiedenen Wissenshintergründen. Die Rolle der ersten muslimischen Gemeinde bzw. Hörerschaft bei der Entwicklung des Korantextes, die Rede von der Gemeindebildung und die verschiedenen Wissenshintergründe der Gemeinde werden hier klargestellt und kritisch diskutiert. Ich beschränke mich in diesem vorliegenden Beitrag in erster Linie auf die Behandlung dieser oben genannten Faktoren bei Neuwirth.

\section{Der Koran als Text der Gemeindebildung bei Neuwirth}

Oben wird von zwei voneinander unterschiedlichen europäischen Richtungen der Koranforschung gesprochen, der traditionellen und der neueren. Die neuere europäische Koranforschung vertreten unter anderem Neuwirth und ihr im Jahr 2007 an der Berlin-Brandenburgischen Akademie der Wissenschaften begonnenes Vorhaben Corpus Coranicum. ${ }^{9}$ Neuwirth missbilligt die traditionelle westliche Koranforschung. Sie richtet ihre Kritik zum Beispiel an Abraham Geiger, der Muhammad allein als Autor des Korans betrachtet und Muhammads Rolle als Verkünder und Ideenverhandler ignoriert. Neuwirth meint, dass der neue Kontext des Korans nicht mehr das arabische Milieu, sondern die spätantike Geisteswelt war. Eine gewisse einseitige Perspektive sei es, die die Rolle der Hörer und deren Einbringung in die Entwicklung des Textes ignoriere und die der steten Verhandlung und der neuen Theologie keine Beachtung

9 Über Neuwirth und das Vorhaben Corpus Coranicum wurde ein Überblick in meiner Dissertation zu Die Methode Nöldekes zur chronologischen Anordnung der Suren und Verse des Korans in seinem Buch „Geschichte des Qorāns. Eine analytisch-kritische Studie gegeben. In dieser vorliegenden Arbeit konzentriere ich mich vor allem auf die Behandlung der Idee, der zufolge der Koran als Text der Gemeindebildung anzusehen ist. 
Ein kritischer Blick auf Angelika Neuwirths Koranverständnis

Dr. Tarek lkot

مجلة وادي النيل للاراسات والبحوث الإنسانية والاجتماعية والتزبوية (مجلة علمية محكمة)

schenke. ${ }^{10}$ Der Koran gilt Neuwirth nicht als „eine auktoriale Schrift, sondern als die Mitschrift einer komplexen Faktoren verdankten Religionsgenese.“"11 Der Koran lässt sich gemäß dieser Vorstellung nicht im Rahmen der islamischen Tradition bzw. des historischen Kontextes des arabischen Milieus wahrnehmen, sondern im Rahmen der spätantiken Geisteswelt.

In ihrer Rede von Theodor Nöldeke und seinem Versuch zur Etablierung einer chronologischen Anordnung des Korans sieht Neuwirth auch in Nöldekes Verständnis des Korans als ein auktorialer Text Fehleinschätzungen. Sie begründet ihre Meinung damit, dass Nöldeke eine Interaktion zwischen Verkünder und Hörern nicht beachtet. Er verstehe den Koran als einen Muhammad allein zugeschriebenen Text. ${ }^{12}$ „Die Bildungsvoraussetzungen wie auch die theologischen Positionen der Hörer werden mit Fortschreiten der Verkündigung also diversifizierter.“13 Im Koran finden sich Diskussionen und Verhandlungen, „die keinen Zweifel an der zumindest passiven Mitautorschaft der Gemeinde lassen." "14 Aus dem letzten Zitat lässt sich folgern, dass die ersten Hörer einen großen Beitrag zur Autorschaft des Korans leisteten. Ihr Beitrag war aber passiv bzw. unmittelbar. Diese passive Mitautorschaft der frühen Gemeinde bedeutet die Autorschaft Muhammads. Ohne Zweifel sieht Neuwirth in Muhammad den Urheber des Korans. Sie erkennt die göttliche Herkunft des Korans nicht an. Dazu versteht man, dass die Ideen und der Inhalt dementsprechend dem Propheten Muhammad und seiner Gemeinde zugeschrieben sind. Die Sprache und die Form gehen auf den Propheten Muhammad allein zurück.

${ }^{10}$ Neuwirth, Angelika: Wie entsteht eine Schrift in der Forschung und in der Geschichte? Die hebräische Bibel und der Koran, Mohr Siebeck, Tübingen 2017, S. 25.

${ }^{11}$ Ebd., S. 27.

${ }^{12}$ Neuwirth, Der Koran, Bd. 2/1: Frühmittelmekkanische Suren, S. 33.

${ }^{13}$ Ebd., S. 33.

${ }^{14}$ Ebd., S. 33. 
(ISSN : 2536 - 9555)

Eine Beziehung zwischen dem Verkünder und seinen Hörern findet man nach Neuwirth im Korantext. Der Koran entsteht dieser Vorstellung zufolge nicht in einem leeren, kulturell isolierten Raum. Die ersten Hörer seien Personen gewesen, die verschiedenen spätantiken Traditionen angehört hätten. Man müsse annehmen, dass die Hörerschaft Muḥammads sich mit vielen biblischen und post-biblischen Traditionen ausgekannt habe. Unmittelbar seien sie aber ausführlich verhandelt, nicht unmittelbar übernommen worden. ${ }^{15}$ Die Idee, der zufolge der Koran in einem arabischen von den anderen Kulturen isolierten Raum entstand, findet bei Neuwirth keine Aufnahme. Der Koran gehört zur Zeit der Spätantike mit all deren Traditionen. Neuwirth bestätigt immer wieder die Bekanntschaft der Gemeinde um Muhammad mit biblischen und post-biblischen Traditionen, die der Verhandlung durch Muhammad und seine Gemeinde unterzogen sind. Die Abhängigkeit des Korans von jüdischen und christlichen Traditionen ist in Neuwirths Worten leicht zu bemerken.

Neuwirth erklärt die Art und Weise der Verhandlung sowie die verhandelten Traditionen. „Der Koran reflektiert den Prozess rigoroser Prüfung, Revision und letztendlich Supersession von grundlegenden jüdischen und christlichen, aber auch paganen Traditionen." ${ }^{\text {"16 }}$ Geprüft, revidiert und ersetzt werden nicht nur jüdische und christliche Traditionen, sondern auch pagane. Dem Koran lagen dieser Ansicht nach verschiedene Quellen, jüdische, christliche und altarabische Traditionen, zu Grunde.

Neuwirth weist darauf hin, dass jüdische, christliche und pagane Traditionen von Muhammad und seiner Gemeinde verhandelt wurden. Es ist hier bemerkenswert, dass ein Unterschied zwischen der traditionellen und neueren Perspektive im Hinblick auf den Koran in der Art und Weise der Übernahme von jüdischen, christlichen und paganen Traditionen liegt. Während die traditionelle Koranforschung davon ausgeht, dass Muhammad allein diese Traditionen auf eine mittelbare Weise

${ }^{15}$ Neuwirth, Koranforschung - eine politische Philologie?, S. 8-9.

${ }^{16}$ Ebd., S. 9. 
Ein kritischer Blick auf Angelika Neuwirths Koranverständnis

Dr. Tarek lkot

مجلة وادي النيل للاراسات والبحوث الإنسانية والاجتماعية والتريوية (مجلة علمية محكمة)

aufnahm, spricht die neuere Koranforschung von Verhandlung dieser Traditionen durch Muhammad und seine Gemeinde.

Den Grund für die neue Wahrnehmung der jüdischen und christlichen Traditionen durch die Gemeinde erläutert Neuwirth im Folgenden: Die koranische Gemeinde habe die Bibel neu wahrgenommen, um ihre eigene Identität zu bilden. Man müsse aber von einer doppelten Bibelrezeption sprechen, einer jüdischen und einer christlichen. Da beide Lektüren der Bibel zur Zeit der Koranentstehung bestanden, stellt Neuwirth die Frage, ob der Koran der jüdischen oder der christlichen Tradition folgt. ${ }^{17}$ Islamisch gesehen folgt der Koran weder jüdischer noch christlicher Tradition. Denn er erscheint der islamischen Überzeugung entsprechend als ein Buch göttlichen Ursprungs.

Im Folgenden erklärt Neuwirth, dass die biblischen Traditionen nicht schriftlich vermittelt wurden, sondern mündlich durch gebildete Personen. Bei der Prüfung des Korans im Hinblick auf seine Bibelrezeption müsse man den Horizont weiten. Die biblischen Traditionen seien mündlich „durch synkretistisch Gebildete in Mekka und jüdisch bzw. christlich Gebildete in Medina“"18 vermittelt worden. Die Rolle des Propheten und seiner Gemeinde als realer Akteure müsse auch wahrgenommen werden. Der Grund dafür besteht darin, dass sie „durch stete Verhandlung nicht nur der biblischen Traditionen, sondern auch noch anderer Formen des kulturellen Erbes, vor allem der paganen altarabischen Dichtung, eine konsensfähige neue Theologie erarbeiten." ${ }^{\text {"19 }}$ Muhammad und seine Gemeinde nahmen die jüdischen, christlichen und paganen Traditionen neu wahr, um ihre eigene Identität zu bilden. Sie kamen durch ständige Verhandlung solcher Traditionen zur Erarbeitung einer neuen Theologie, deren Wurzeln sich vor allem laut Neuwirth in der Bibel finden.

\footnotetext{
${ }^{17}$ Ebd., S. 9-10.

${ }^{18}$ Ebd., S. 17.

${ }^{19}$ Ebd., S. 17.
} 
(ISSN : 2536 - 9555)

Genau wie Geiger, Nöldeke und andere europäische Koranforscher behauptet Neuwirth, die biblischen Traditionen seien mündlich wiedergegeben worden. ${ }^{20}$ Während Geiger, Nöldeke und andere europäische Koranforscher nur Muhammad die Übernahme biblischer Traditionen aus mündlichen Überlieferungen bzw. aus Gesprächen mit Juden und Christen zuschreiben, spricht Neuwirth von der Gemeinde bei mündlicher Wiedergabe der biblischen Traditionen. Die Gemeinde bestand ihrer Auffassung nach aus jüdisch bzw. christlich Gebildeten.

Neuwirth betont den engen Bezug des Korans zur Bibel, beschreibt den Koran als „,die neue arabische Deutung der Bibel ${ }^{\text {‘2 } 1}$ und betrachtet Muhammad als einen arabischen Propheten und einen aktuellen Ausleger der biblischen Traditionen. Die Hörer seien spätantike Gebildete, am ehesten Synkretisten, die die wichtigsten jüdischen und christlichen Traditionen gekannt hätten. ${ }^{22}$

Bei Neuwirth liest man immer wieder den Begriff „Spätantike“. „Was bedeutet „Spätantike“ bezogen auf den Koran?“ Dass der Koran als der islamische Text schlechthin beschrieben werde, widerspräche dem Sachverhalt. Der Koran sei ein Spiegel der spätantiken theologischen Traditionen. Spätantike verstehe sich als ein Denkraum, in dem verschiedene antike Traditionen neu gelesen würden. ${ }^{23}$ Der Koran gelte deshalb als ein Text, „der mehrere „Antiken“, die biblische, die pagan-arabische, gelegentlich sogar die hellenistische, einer neuen Interpretation unterwirft. .24

20 Elkot, Tarek Anwar Abdelgayed: Die Methode Nöldekes zur chronologischen Anordnung der Suren und Verse des Korans in seinem Buch „Geschichte des Qorāns. Eine analytisch-kritische Studie, Göttingen 2014. [online publizierte Dissertation], S. 62.

${ }^{21}$ Neuwirth, Koranforschung - eine politische Philologie?, S. 14.

${ }^{22}$ Neuwirth, Wie entsteht eine Schrift in der Forschung und in der Geschichte?, S. 111.

${ }^{23}$ Neuwirth, Koranforschung - eine politische Philologie?, S. 45.

${ }^{24}$ Ebd., S. 45. 
Ein kritischer Blick auf Angelika Neuwirths Koranverständnis

Dr. Tarek lkot

مجلة وادي التيل للاراسات والبحوث الإنسانية والاجتماعية والتربوية (مجلة علمية محكمة)

Die Interaktion zwischen Verkünder und Gemeinde, die sich auf den Koran auswirkt, umfasse nicht nur die theologischen Hintergründe, die im Koran verhandelt worden seien, sondern auch die Sprachform. Die Sprachgestalt des Korans sei nicht nur Ausdruck des arabischen Kulturraums, ,sie reflektiert in ihrer besonderen rhetorischen Form zugleich das Szenario einer Interaktion zwischen Prophet und Hörern. “.25

\section{Beispiele für die Betrachtung des Korans als Text der Gemeindebildung}

In den Suren fände sich wiederholte Rezitation, was auf die Hinzufügung neuer Einsichten hinweise. Die volle theologische Dimension biblischer Geschichten sei erst bei wiederholter Rezitation offenkundig geworden, die eventuell die Hörer aufweisen würden, die in der biblischen Tradition gebildet gewesen seien. Was sich im Laufe der Zeit verändere, seien die Hörer, die aus verschiedenen Gruppen bestünden und die zu neuen Einsichten kämen. Es handle sich bei der Verkündigung nicht nur um Texte, sondern um die Träger, die in der biblischen Tradition gebildet gewesen seien. ${ }^{26}$ Neuwirth versucht wiederum die Abhängigkeit des Korans von der Bibel respektive die Beeinflussung des Korans durch die jüdischen und christlichen Traditionen zu bestätigen. Durch die ersten Hörer, die jüdische und christliche Gebildete waren, wurden die jüdischen und christlichen Traditionen auf den Koran übertragen. Die Entwicklung des Korantextes ist demgemäß mit der Entwicklung der Hörer eng verbunden. Neuwirth spricht hier über die biblischen Erzählungen im Koran als ein Beispiel dafür. Bemerkenswert ist, dass der Prophet Muhammad als wichtiger Akteur nicht angeführt wird. Sie verbindet diese in den Geschichten beobachtete Entwicklung mit

${ }^{25}$ Neuwirth, Der Koran, Bd. 2/1: Frühmittelmekkanische Suren, S. 38.

${ }^{26}$ Neuwirth, Wie entsteht eine Schrift in der Forschung und in der Geschichte?, S. 85, 87. 
(ISSN : 2536 - 9555)

der in der jüdischen und christlichen Tradition gebildeten Gemeinde.

Unter dem Titel Zwei kongeniale Propheten: Mose und Muhammad im Buch Wie entsteht eine Schrift in der Forschung und in der Geschichte? Die hebräische Bibel und der Koran spricht Neuwirth davon, dass Mose zum Modell für Muhammad wird und biblisches Wissen übernommen wird, aber der Prozess geschieht nicht linear-einseitig, sondern auch rückläufig. Neuwirth schreibt der Hörergemeinde die Umformung älterer Traditionen zu. Die Gemeinde gibt den Traditionen aber ihr eigenes arabisches spätantikes Gepräge. ${ }^{27}$ Neuwirth macht hier den Propheten Muhammad für die Umformung älterer Traditionen nicht verantwortlich, sondern die Gemeinde. Sie legt zu viel Gewicht auf die Gemeinde.

Als Beispiel für die neue Verhandlung der christlichen Tradition zu Maria und Jesus thematisiert Neuwirth Sure 19, Maria. ${ }^{28}$ Es erweise sich der Text von Sure 19 ,als Gespräch zwischen verschiedenen Traditionen. ${ }^{“ 29}$ Die Geschichte von Maria stehe mit dem apokryphen Protevangelium des Jakobus im Einklang. ${ }^{30}$ In diesem Zusammenhang stellt sich die Frage nach dem Grund für die Benutzung der Stoffe über die Geschichte von Maria und Jesus aus nichtkanonischen biblischen Schriften und die Nichtbenutzung der Stoffe aus kanonischen biblischen Schriften. War ferner das apokryphe Protevangelium des Jakobus der ersten islamischen Gemeinde vertraut.

Die Gebetsrichtung nach Jerusalem verbindet Neuwirth ebenso nicht mit einer neuen Stellung Muhammads zur Wendung im Gebet, sondern mit der Gemeinde. „Mit dieser Gebetsrichtung bringt die frühe Gemeinde des Verkünders jedenfalls symbolisch ihre Zugehörigkeit zur biblischen Tradition zum Ausdruck. ${ }^{\text {(31 }}$ Die

${ }^{27}$ Ebd., S. 99.

${ }^{28}$ Neuwirth, Der Koran als Text der Spätantike, S. 453.

${ }^{29}$ Neuwirth, Der Koran, Bd. 2/1: Frühmittelmekkanische Suren, S. 645.

${ }^{30}$ Ebd., S. 645-646.

${ }^{31}$ Neuwirth, Der Koran als Text der Spätantike, S. 468. 
Ein kritischer Blick auf Angelika Neuwirths Koranverständnis

Dr. Tarek lkot

مجلة وادي النيل للاراسات والبحوث الإنسانية والاجتماعية والتربوية (مجلة علمية محكمة)

Zugehörigkeit der frühen Gemeinde zur biblischen Tradition bedeutet nach diesem Verständnis die Zugehörigkeit des Korans zur jüdischen und christlichen Tradition.

Im Hinblick auf das Erscheinen neuer Begriffe im Koran wie muhkam (eindeutig) bzw. mutashābih (mehrdeutig) spricht Neuwirth von einer Hypothese, der zufolge Ideen zwischen der Gemeinde und den Juden von Medina ausgetauscht werden. ${ }^{32}$ Daraus wird ersichtlich, dass nicht nur theologische Traditionen durch die jüdisch oder christlich gebildete Gemeinde in den Koran eingedrungen sind, sondern auch neue Begriffe.

Was die im Zentrum der Sure 3 stehende Erzählung das Haus Abraham und das Haus Amram betrifft, schließt Neuwirth den Austausch der Gemeinde mit den medinischen Juden aus. Hier sollen christliche Traditionselemente der Gemeinde bekannt gewesen sein. ${ }^{33}$ Neuwirth schließt den jüdischen Einfluss aus, nimmt aber den christlichen an, ohne dies wissenschaftlich zu begründen. Darüber hinaus spricht sie dem Propheten Muhammad eine Rolle ab.

Als ein weiteres Beispiel dafür, dass der Koran spätantike christliche apokryphe Traditionen bearbeitet bzw. ihnen eine neue theologische Position gibt, liefert Michael Marx die Geschichte des Vogelwunders Jesu und zieht einen Vergleich zwischen dem koranischen Text und dem Kindheitsevangelium des Thomas. Seiner Meinung nach war Texttradition Anfang des siebten Jahrhunderts in Mekka und Medina der ersten islamischen Gemeinde bekannt. ${ }^{34}$ Marx begründet nicht, warum der Koran sich

${ }^{32}$ Ebd., S. 532.

${ }^{33}$ Ebd., S. 532-533.

${ }^{34}$ Marx, Michael J.: Ein Koran-Forschungsprojekt in der Tradition der Wissenschaft des Judentums: Zur Programmatik des Akademievorhabens Corpus Coranicum, in: Hartwig, Dirk [u.a.] (Hrsg.): „Im vollen Licht der Geschichte,.. Die Wissenschaft des Judentums und die Anfänge der kritischen Koranforschung, Ergon Verlag, Würzburg 2008, S. 49. 
(ISSN : 2536 - 9555)

an spätantike christliche apokryphe Traditionen anlehnt oder warum die Gemeinde diese apokryphen Traditionen heranzieht. War die Gemeinde nur mit den christlichen apokryphen Traditionen über die Geschichte des Vogelwunders Jesu vertraut? Kann man nachweisen, dass die in den Kanon aufgenommenen, echten biblischen Traditionen der ersten islamischen Gemeinde nicht zugänglich waren?

Die ganze Darstellung und die oben genannten Beispiele bestätigen Neuwirths These, der zufolge die in den biblischen Traditionen gebildete Gemeinde einen bedeutenden Beitrag zur passiven Autorschaft des Korans leistete. Diese passive Autorschaft setzt meiner Ansicht nach eine höhere religiöse Bildung der Gemeinde voraus, die Bekanntschaft der Gemeinde in Mekka und Yatrib mit biblischen und post-biblischen Traditionen, einen engen Umgang der Gemeinde mit Juden und Christen und nicht zuletzt die Verbreitung der jüdischen und christlichen Gedanken in Mekka und Yatrib. Andere Quellen, vor allem arabische, die von Neuwirth nicht berücksichtigt werden, enthalten andere Aussagen.

Nach Ğawād 'Alī geht aus dem Koran hervor, dass der direkte Kontakt des Propheten Muhammad mit den Juden in Yatrib begann. In Mekka seien die Juden kaum von Bedeutung gewesen. Dies dokumentiere sich deutlich in den jeweiligen mekkanischen und medinensischen Suren. Die Juden hätten in Mekka weder einen großen Einfluss gehabt noch große Gemeinden gebildet. Wenn dies der Fall gewesen wäre, hätte man davon erfahren, hätten sie ein eigenes Viertel und einen Spitzenplatz unter den Leuten aus dem Stamm der Quraiš gehabt und der Koran hätte darauf in den mekkanischen Suren Bezug genommen. Im Gegensatz dazu hätten die Juden, die einen engen Bezug zu den beiden Stämmen Aus und Hazrağ gehabt hätten, in Yatrib gelebt. Von ihnen sprächen die medinensischen Suren. ${ }^{35}$

35 'Alī, Ğawād: Al-mufașṣal fì tārīh al- 'arb qabl al-Islām, 4. Aufl., Dār as-Sāqī, o. O. 2001, Bd. 12, S. 119. 
Ein kritischer Blick auf Angelika Neuwirths Koranverständnis

Dr. Tarek lkot

مجلة وادي النيل للاراسات والبحوث الإنسانية والاجتماعية والتزبوية (مجلة علمية محكمة)

Julius Wellhausen (1844-1948) zählt die Oasen des Nordwestens des Arabien, Taima, Chaibar, Jathrib und Fadak, zu den Orten, die die Juden in Besitz genommen hatten. ${ }^{36}$ Daraus lässt sich entnehmen, dass Mekka nicht zu den Orten gehörte, in dem die Juden sich niederließen. Woher hatte die islamische Gemeinde in Mekka die jüdischen Ideen? Oder wie gelangten die jüdischen Ideen an den Propheten Muhammad und seine Gemeinde in Mekka?

Über die Art und Weise des Lebens der Juden meint Wellhausen, dass die Juden, in ihren eigenen geschlossenen Gemeinden ${ }^{\text {“37 }}$ lebten. Sie hätten sich nicht für die Verbreitung des Judentums unter den Arabern interessiert. ${ }^{38}$ Dass die Juden in geschlossenen Gemeinden lebten und sich nicht mit der Verbreitung des Judentums beschäftigten, erscheint mir als ein Beleg dafür, dass die jüdischen Ideen den Arabern nicht sehr vertraut waren.

Wellhausen thematisiert auch die Verbreitung des Christentums unter den Stämmen in Arabien. Uns interessiert in diesem Zusammenhang vor allem der Einfluss des Christentums in Hiğāz und Zentralarabien. Dazu schreibt er Folgendes:

„Im Hiğāz und in Centralarabien war es allerdings nicht so weit. Die dort wohnhaften Mudarstämme bewahrten das alte heidnische Wesen treuer und waren dem aramäischen Einflusse weniger zugänglich als die Stämme an der Grenze; dadurch erklärt sich vielleicht zum Teil ihre Entfremdung von den Rabī'a und Taiji und besonders von den Qudā'a. Aber auch bei ihnen sickerte das Christentum ein, durch Sklaven, durch den Verkehr, durch den Handel.“" ${ }^{\text {39 }}$

\footnotetext{
${ }^{36}$ Wellhausen, Julius: Reste arabischen Heidentums, Gesammelt und Erläutert, 3., unveränderte Aufl., Walter de Gruyter, Berlin 1961, S. 230.

${ }^{37}$ Ebd., S. 230.

${ }^{38}$ Ebd., S. 230.

${ }^{39}$ Ebd., S. 231.
} 
(ISSN : 2536 - 9555)

Über die Verbreitung der christlichen Lehren und Riten in Mekka formuliert Wellhausen Folgendes:

„Eine oberflächliche Kenntnis der christlichen Einrichtungen, Riten und Lehren, auch wol mancher Legenden und biblischen Geschichten war z. B. in Mekka ohne Zweifel verbreitet; die dortigen Kaufleute schöpften sie einerseits in Habesch und im Jaman, andererseits in Syrien und im Irāq auf ihren Handelsreisen. “40

Die Worte Wellhausens weisen darauf hin, dass es sich dabei um oberflächliche Kenntnisse der christlichen Lehren und Gebräuche handelte, die durch die Handelsreisen nach Mekka gebracht worden waren. Wie hätte sich die islamische Gemeinde in Mekka, die über oberflächliche bzw. geringe Kenntnisse des Christentums verfügt haben sollte, an der Autorschaft eines Textes wie des Korans beteiligen können? Kann plausibel nachgewiesen werden, dass die islamische Gemeinde in Mekka jüdisch und christlich gebildet war?

In seiner Rede von der Gelehrsamkeit und der Bildung der damaligen Araber meint Ibn Haldūn, dass die Araber weder Schrift noch Gelehrsamkeit besaßen. Sie seien Nomaden und Analphabeten gewesen. Wenn sie sich danach gesehnt hätten, etwas zu wissen, hätten sie die Schriftbesitzer (die Juden und die Christen) gefragt. Was die Gelehrsamkeit und die Bildung der Juden angeht, vertritt Ibn Haldūn auch die Ansicht, dass die Juden, die zu dieser Zeit unter den Arabern waren, auch Nomaden gewesen waren. Sie hätten nichts mehr außer dem gewusst, was die einfachen Schriftbesitzer gewusst hätten. ${ }^{41}$ Im Gegensatz zu den

${ }^{40}$ Ebd., S. 231-232.

41 Ibn Haldūn, 'Abd ar-Raḥmān b. Muhammad: Tārīh Ibn Haldūn. Dīwān al-mubtad' wa-l-habar fì aiyām al- 'arab wa-l- 'ağam wa-l-barbar wa-man 'āṣarāhum min dawi aš-ša'n al-akbar, ediert von Hुalīl Šahāâ,a, 2. Aufl., Dār al-fikr, Beirut 1988, Bd. 1., S. 554-555. 
Ein kritischer Blick auf Angelika Neuwirths Koranverständnis

Dr. Tarek lkot

$$
\text { مجلة وادي التيل للاراسات والبحوث الإنسانية والاجتماعية والتربوية (مجلة علمية محكمة) }
$$

Juden anderer Länder war der Grad der Bildung von der Mehrheit der Juden auf der arabischen Halbinsel gering. ${ }^{42}$

Die geringe Bildung bzw. die niedrige Gelehrsamkeit in Arabien machen die Möglichkeiten eines Beitrags an der Urheberschaft des Korans vonseiten der damaligen islamischen Gemeinde eigentlich unmöglich.

Überdies muss hier darauf aufmerksam gemacht werden, dass die zusammen mit der frühen islamischen Gemeinde im gleichen Milieu lebenden Ungläubigen in einigen Koranversen aufgefordert wurden, zehn Suren oder eine Sure gleicher Art des Korans beizubringen. ${ }^{43}$ Ginge der Koran auf den Propheten Muhammad und seine Gemeinde zurück, wie Neuwirth meint, hätten die ungläubigen, einfach gebildeten Menschen einen Text gleicher Art des Korans verfassen können. Dass sie dies nicht zu tun vermochten, unterstützt den Glauben an den göttlichen Ursprung des Korantextes.

\section{Fazit}

Die neuere Koranforschung in Europa fokussiert ihr Interesse auf die erste islamische Gemeinde als sehr wichtigen Akteur bei der Entstehung des Korans. In ihren verschiedenen Werken und Artikeln über den Koran versucht Neuwirth wiederholt zu bestätigen, dass der Koran kein auktorialer Text ist. Den Koran dem Propheten Muhammad allein zuzuschreiben, wie Vertreter der traditionellen europäischen Koranforschung meinen, weist Neuwirth zurück. Der Koran gilt ihr als ein Text, an dessen Konstitution sich verschiedene Akteure beteiligen. Die Gemeinde um Muhammad spielt nach Neuwirth eine sehr wichtige und

42 'Alī, Al-mufașșal fì tārīh al- 'arb qabl al-Islām, Bd. 12, S. 133; Nöldeke, Theodor: Geschichte des Qorāns, bearbeitet von Friedrich Schwally, 2. Auflage, Dieterich'sche Verlagsbuchhandlung, Leipzig 1909, Bd.1, S. 169.

${ }^{43}$ (Sure 2, Koranvers 23), (Sure 11, Koranvers 13) und (Sure 10, Koranvers 38). 
(ISSN : 2536 - 9555)

unerlässliche Rolle dabei. Deshalb betrachtet Neuwirth den Koran nicht als Dokument einer einzelnen Person, sondern als Dokument einer Gemeindebildung.

Die Gemeinde um Muhammad ist Neuwirth zufolge jüdisch und christlich gebildet. Daher enthält der Koran jüdische und christliche Traditionen, die aber nicht unmittelbar übernommen, sondern durch Muhammad und seine Gemeinde verhandelt werden. Neben jüdischen und christlichen Traditionen finden sich nach Neuwirth ebenso pagane.

Damit betont sie den Bezug des Korans zur Bibel bzw. die Abhängigkeit des Korans von den jüdischen und christlichen Traditionen. Sie beschreibt den Koran darum als die neue arabische Deutung der Bibel und Muhammad als einen arabischen Propheten und einen aktuellen Ausleger der biblischen Traditionen.

Diesem Verständnis entsprechend beschränkt sich die Rolle der frühen Gemeinde nicht nur auf die Debatten und Diskussionen mit dem Verkünder, sondern auch auf die passive Mitautorschaft des Korans. Sie wird neben dem Propheten Muhammad als Verfasserin des Korans betrachtet. Zusammen mit dem Verkünder verhandelte die Gemeinde mittels Prüfung und Revision jüdische, christliche und pagane Traditionen.

Meiner Meinung nach ist kein qualitativer Unterschied zwischen der traditionellen und neueren europäischen Koranforschung im Hinblick auf die Auffassung zum Ursprung des Korans zu sehen. Genauso wie die Vertreter der traditionellen Koranforschung spricht Neuwirth als Vertreterin der neueren Koranforschung dem Koran seinen göttlichen Ursprung ab. Der Koran ist ihr zufolge ein menschlicher Text, der nicht nur auf Muhammad zurückzuführen ist, sondern auch auf die Gemeinde.

Nach islamischer Auffassung bringt der Koran weder einen auf den Propheten Muhammad zurückgehenden Text noch die religiöse Bildung bzw. die jüdischen, christlichen und altarabischen Hintergründe der frühen islamischen Gemeinde zum Ausdruck. Er ist ein dem Propheten Muḥammad offenbarter Text. 
Ein kritischer Blick auf Angelika Neuwirths Koranverständnis

Dr. Tarek lkot

مجلة وادي النيل للاراسات والبحوث الإنسانية والاجتماعية والتربوية (مجلة علمية محكمة)

\section{Literatur}

'Alī, Ğawād: Al-mufașṣal fì tārīh al-'arb qabl al-Islām, 4. Aufl., Dār as-Sāqī, o. O. 2001.

Elkot, Tarek Anwar Abdelgayed: Die Methode Nöldekes zur chronologischen Anordnung der Suren und Verse des Korans in seinem Buch „Geschichte des Qorāns. Eine analytischkritische Studie, Göttingen 2014. [online publizierte Dissertation].

Ibn Ḩaldūn, 'Abd ar-Raḥmān b. Muḥammad: Tārīh Ibn Haldūn. Dīwān al-mubtad' wa-l-habar fì aiyām al- 'arab wa-l- 'ağam wa-l-barbar wa-man 'āṣarāhum min dawī aš-ša'n al-akbar, ediert von Ḩalīl Šahāāa, 2. Aufl., Dār al-fikr, Beirut 1988.

Khorchide, Mouhand: Gottes Offenbarung in Menschenwort, Der Koran im Licht der Barmherzigkeit, Herder Verlag, Freiburg 2018.

Marx, Michael J.: Ein Koran-Forschungsprojekt in der Tradition der Wissenschaft des Judentums: Zur Programmatik des Akademievorhabens Corpus Coranicum, in: Hartwig, Dirk [u.a.] (Hrsg.): „Im vollen Licht der Geschichte“. Die Wissenschaft des Judentums und die Anfänge der kritischen Koranforschung, Ergon Verlag, Würzburg 2008.

Neuwirth, Angelika: Der Koran als Text der Spätantike. Ein europäischer Zugang, 1. Aufl., Verlag der Weltreligionen, Berlin 2010.

Neuwirth, Angelika: Der Koran, Bd. 1: Frühmekkanische Suren, Poetische Prophetie, Handkommentar mit Übersetzung von Angelika Neuwirth, 1. Aufl., Verlag der Weltreligionen, Berlin 2011. 
(ISSN : 2536 - 9555)

Neuwirth, Angelika: Koranforschung - eine politische Philologie? Bibel, Koran und Islamentstehung im Spiegel spätantiker Textpolitik und moderner Philologie, De Gruyter, Berlin 2014.

Neuwirth, Angelika: Der Koran, Bd. 2/1: Frühmittelmekkanische Suren, Das neue Gottesvolk: Biblisierung des altarabischen Weltbildes, Handkommentar mit Übersetzung von Angelika Neuwirth, 1. Aufl., Verlag der Weltreligionen, Berlin 2017.

Neuwirth, Angelika: Wie entsteht eine Schrift in der Forschung und in der Geschichte? Die hebräische Bibel und der Koran, Mohr Siebeck, Tübingen 2017.

Nöldeke, Theodor: Geschichte des Qorāns, bearbeitet von Friedrich Schwally, 2. Auflage, Dieterich'sche Verlagsbuchhandlung, Leipzig 1909.

Özsoy, Ömer: Das Unbehagen der Koranexegese - Den Koran in anderen Zeiten zum Sprechen bringen, in: Kontexte, Methoden, Inhalte, Frankfurter Zeitschrift für Islamischtheologische Studien, EB-Verlag, Berlin 2014.

Wellhausen, Julius: Reste arabischen Heidentums, Gesammelt und Erläutert, 3., unveränderte Aufl., Walter de Gruyter, Berlin 1961.

Würsch, Renate: Der Koran und seine Rezeption, in: Das Mittelalter 2018 (2013) 1, S. 27-45. 